\title{
Magnetic Properties of Epitaxial (Ge,Mn)Te Thin Films with Varying Crystal Stoichiometry
}

\author{
W. $\mathrm{KnOFF}^{a, *}, \mathrm{~V}$. Domukhovski ${ }^{a}, \mathrm{~K} . \mathrm{DyBko}^{a}, \mathrm{P} . \mathrm{DziAWA}^{a}$, \\ M. Górska ${ }^{a}$, R. Jakięa ${ }^{a}$, E. Eusakowska ${ }^{a}$, A. ReszkA $^{b}$, \\ B. Taliashvili ${ }^{a}$, T. Story ${ }^{a}$, J.R. Anderson ${ }^{c}$ \\ AND C.R. RotUndu ${ }^{c, \dagger}$ \\ ${ }^{a}$ Institute of Physics, Polish Academy of Sciences \\ al. Lotników 32/46, 02-668 Warsaw, Poland \\ ${ }^{b}$ Faculty of Mathematics and Natural Sciences, College of Science \\ Cardinal S. Wyszyński University, Dewajtis 5, 01-185 Warsaw, Poland \\ ${ }^{c}$ Department of Physics and Center for Nanophysics and Advanced Materials \\ University of Maryland, College Park, MD 20742, USA
}

\begin{abstract}
Magnetization of $1 \mu \mathrm{m}$ thick ferromagnetic IV-VI (Ge,Mn)Te semiconductor layers with 10 at.\% of Mn was studied by SQUID magnetometry method up to the magnetic fields of $70 \mathrm{kOe}$. The layers were grown on $\mathrm{BaF}_{2}$ (111) substrates by molecular beam epitaxy with varying Te molecular flux, which permitted the control of layer stoichiometry and conducting hole concentration. X-ray diffraction and in situ electron diffraction characterization of layer growth and crystal structure revealed two-dimensional mode of growth and monocrystalline rhombohedral crystal structure of (Ge,Mn) Te layers. Controlling the layer stoichiometry influences the temperature dependence of magnetization with the ferromagnetic Curie temperature varying in $\mathrm{Ge}_{0.9} \mathrm{Mn}_{0.1}$ Te layers from $T_{\mathrm{c}}=30 \mathrm{~K}$ (low Te flux) to $T_{\mathrm{c}}=42 \mathrm{~K}$ (high $\mathrm{Te}$ flux).
\end{abstract}

PACS numbers: 75.50.Pp

\section{Introduction}

(Ge,Mn)Te polycrystals were among the first diluted magnetic (semimagnetic) semiconductors in which ferromagnetism was discovered and explained by the indirect exchange interaction via the spin polarization of conducting carri-

\footnotetext{
*corresponding author; e-mail: knoff@ifpan.edu.pl

${ }^{\dagger}$ Present address: Material Sciences Division, Lawrence Berkeley National Laboratory, Berkeley, CA 94720, USA.
} 
ers (the Ruderman-Kittel-Kasuya-Yosida (RKKY) mechanism) [1]. In the IVVI family of diluted magnetic semiconductors, based on the alloys of GeTe, $\mathrm{SnTe}$ or PbTe with Mn, a very high concentration of conducting holes ( $p=$ $10^{19}-10^{21} \mathrm{~cm}^{-3}$ ) is typically present. The high value of $p$ is due to the characteristic of these materials to grow non-stoichiometrically with the creation of electrically active native defects (cation vacancies) thus establishing a metallic type of electrical conductivity. Mn ions in these materials are known to be in $\mathrm{Mn}^{2+}$ charge state with the electronic configuration $3 d^{5}$ creating the well localized spin-only magnetic moments of $5 \mu_{\mathrm{B}}$ (the Bohr magnetons) [2]. As Mn ions in IV-VI semiconductors are electrically neutral, one can independently control the concentration of carriers and magnetic ions content by applying, e.g., a simple annealing procedure. The experimental studies of the magnetic properties of $(\mathrm{Pb}, \mathrm{Sn}, \mathrm{Mn}) \mathrm{Te}$ and $(\mathrm{Sn}, \mathrm{Mn}) \mathrm{Te}$ carried out in a broad range of conducting hole concentration and Mn ion content revealed that both the RKKY ferromagnet and the RKKY spin glass state are observed in IV-VI semiconductors with Mn [2-4].

Recent advances in the growth of high quality $(\mathrm{Ge}, \mathrm{Mn}) \mathrm{Te}$ layers by ionized cluster beam and molecular beam epitaxy (MBE) as well as by sputtering extended the accessible range of Mn content and hole concentration available with the maximum ferromagnetic Curie temperature about $T_{\mathrm{c}}=150-200 \mathrm{~K}$ for $\mathrm{Mn}$ content of 40 at.\% [5-8]. In the epitaxial layers of (Ge,Mn)Te the control of carrier concentration can be efficiently achieved by the proper choice of growth conditions. To fully explore these new technological possibilities in the studies of magnetic properties of diluted ferromagnetic semiconductors one has to examine magnetic, structural, and electrical properties of the layers grown under various technological regimes.

In this work, the influence of crystal stoichiometry on the magnetic properties of monocrystalline $(\mathrm{Ge}, \mathrm{Mn}) \mathrm{Te}$ layers grown by MBE is experimentally studied by the superconducting (SQUID) magnetometry method. The set of $(\mathrm{Ge}, \mathrm{Mn}) \mathrm{Te}$ layers was grown at the same technological conditions with Mn content of 10 at.\% but with systematically varying additional Te flux applied during the MBE growth in order to facilitate the control of the concentration of native defects (Ge vacancies introducing conducting holes) and the degree of electronic disorder present in these alloys.

\section{Growth and structural characterization of layers}

$(\mathrm{Ge}, \mathrm{Mn}) \mathrm{Te}$ layers with thickness about one micron were grown on freshly cleaved monocrystalline $\mathrm{BaF}_{2}$ (111) substrates at temperature $T_{\mathrm{S}}=280^{\circ} \mathrm{C}$ using a home-built MBE facility. Effusion cells with solid sources of GeTe and Mn were heated up to $T=490^{\circ} \mathrm{C}$ and $T=770^{\circ} \mathrm{C}$, respectively, in order to produce molecular fluxes equivalent to the partial pressures of $P \approx 7 \times 10^{-7} \mathrm{mbar}(\mathrm{GeTe})$ and $P \approx 1.25 \times 10^{-8} \mathrm{mbar}(\mathrm{Mn})$. The additional flux of Te molecules from the separate effusion cell was adjusted for each growth process in the corresponding range of Te partial pressure $P \approx 0.6 \times 10^{-7}-1.9 \times 10^{-7}$ mbar. The in situ reflection high 
energy electron diffraction (RHEED) measurements during the layer deposition process revealed a clear streaky pattern indicating a two-dimensional mode of layer growth. Post-growth $(\mathrm{Ge}, \mathrm{Mn})$ Te layers were annealed at high vacuum in the MBE growth chamber at $T=280^{\circ} \mathrm{C}$ in order to improve their electrical and chemical micro-homogeneity. X-ray diffraction measurements carried out at room temperature revealed good crystal quality of (111)-oriented monocrystalline (Ge,Mn)Te layers with the rocking curve width parameter in the range 200-800 arcsec. The chemical composition analysis performed by energy dispersive X-ray fluorescence (EDXRF) method showed the total Mn content of 10 at.\%, as intended. In all the layers studied the secondary ion mass spectroscopy (SIMS) proved very homogeneous distribution of Ge and Te. Mn composition SIMS profile along the growth direction showed good homogeneity in the volume of the 1 micron thick layer but a substantial reduction of $\mathrm{Mn}$ content in a $0.05-0.1$ micron thick region close to the layer surface. The surface morphology of the layers was studied at room temperature (in air) by atomic force microscopy (AFM) revealing good smoothness of the surface of $(\mathrm{Ge}, \mathrm{Mn}) \mathrm{Te}$ layers with root mean square (RMS) roughness parameter in the range 1-9 $\mathrm{nm}$ for the analysed area of $10 \times 10 \mu \mathrm{m}^{2}$.

\section{Experimental studies of magnetic properties}

The measurements of the temperature and magnetic field dependence of magnetization of $(\mathrm{Ge}, \mathrm{Mn})$ Te layers were performed at low $(H \leq 1 \mathrm{kOe})$ and high $(H \leq 70 \mathrm{kOe})$ magnetic fields using superconducting SQUID magnetometers. All the $(\mathrm{Ge}, \mathrm{Mn})$ Te layers studied undergo at low temperatures a ferromagnetic transition as evidenced by magnetization hysteresis loops presented in Fig. 1a for the (Ge,Mn)Te layers S1-S5 with the same Mn content (10 at.\%) but with crystal stoichiometry varying systematically in a way indicated in this figure as high (sample S1) to low (sample S5) additional Te flux. For these samples the $\mathrm{BaF}_{2}$ contribution has not been subtracted. The Hall effect measurements carried out at temperature $T=77 \mathrm{~K}$ revealed $p$-type conductivity with a very high conducting hole concentration varying in the range $p=(5-10) \times 10^{21} \mathrm{~cm}^{-3}$. In all the layers the low field magnetization measurements indicate that at the applied field of $1 \mathrm{kOe}$ the samples appear to not reach magnetic saturation. In order to study further this effect the magnetization of the $\mathrm{Ge}_{0.9} \mathrm{Mn}_{0.1}$ Te layer grown on $\mathrm{BaF}_{2}$ substrate with the same technological facility was studied for magnetic fields up to $70 \mathrm{kOe}$ (see Fig. 1b). For this sample the $\mathrm{BaF}_{2}$ contribution has been subtracted. The Te flux applied during the growth of this layer was equivalent to partial pressure $P \approx 0.81 \times 10^{-7} \mathrm{mbar}$ (i.e. close to growth regime of the sample S5). We see that at $T=5 \mathrm{~K}$ saturation was not reached even at $70 \mathrm{kOe}$. At higher temperature saturation is less likely. We believe that saturation at such fields would not be found in our other samples.

In Fig. 2a the temperature dependence of magnetization $M(T)$ is shown in the temperature range $T=5-130 \mathrm{~K}$ for the layers S1-S5. For the measurements 

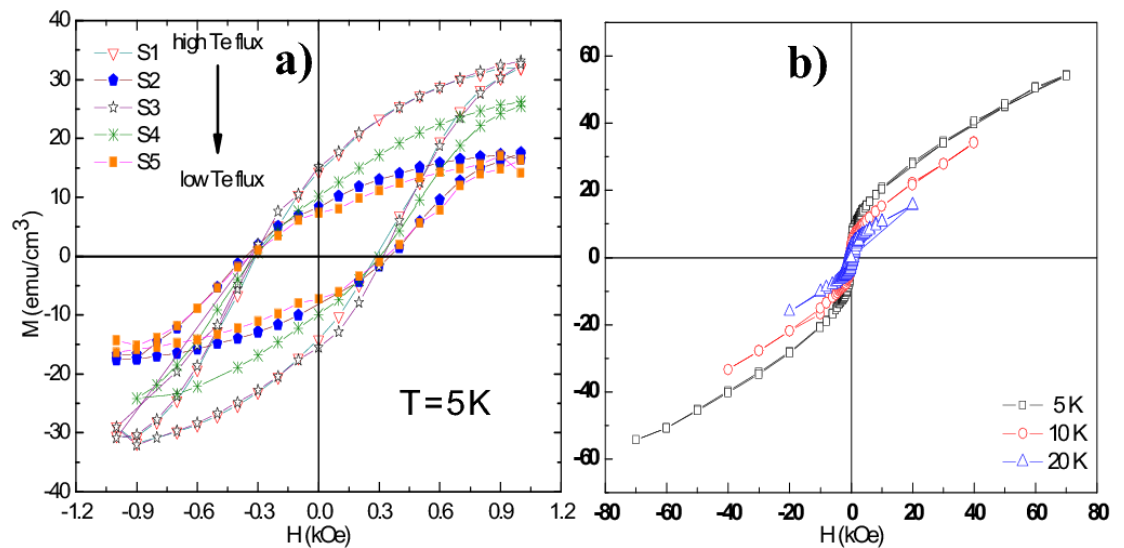

Fig. 1. (a) Magnetization hysteresis loops at low magnetic fields for (Ge,Mn)Te layers with the same Mn content (10 at.\%) but grown under varying Te flux conditions. (b) High field magnetization of $\mathrm{Ge}_{0.9} \mathrm{Mn}_{0.1}$ Te layer with $\mathrm{BaF}_{2}$ contribution subtracted.
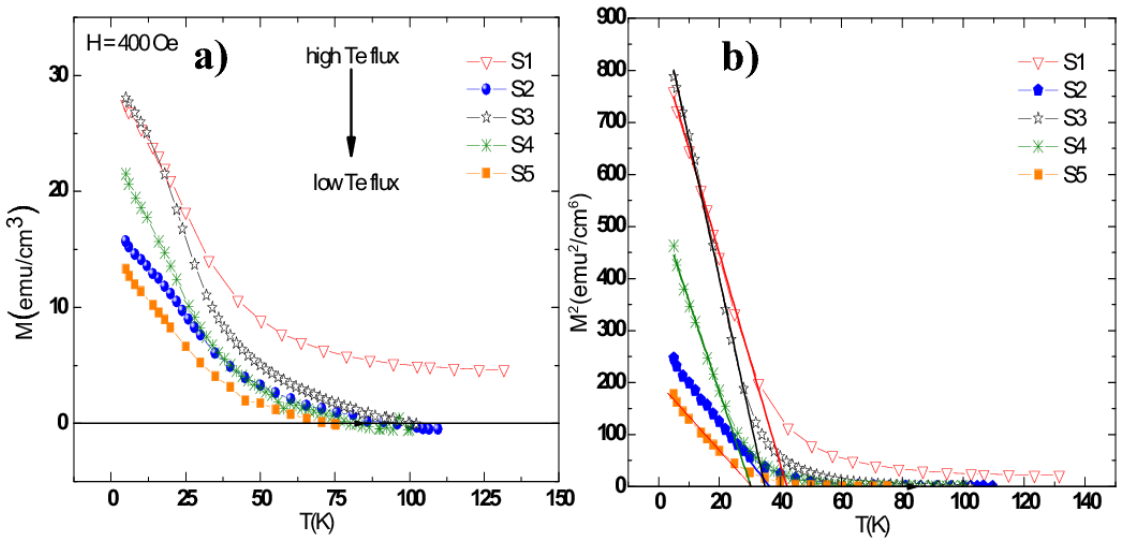

Fig. 2. The temperature dependence of magnetization measured at $400 \mathrm{Oe}$ (a) and the square of magnetization (b) for $\mathrm{Ge}_{0.9} \mathrm{Mn}_{0.1}$ Te layers with varying crystal stoichiometry as controlled by the additional Te flux. The lines show the mean field analysis of $M^{2}(T)$ determining the Curie temperature.

of the $M(T)$ dependence the external magnetic field of $H=400$ Oe was applied along the cleaved [1-10] direction in the (111) layer plane. In Fig. 2b the $M^{2}(T)$ dependence is shown for the same set of data. By extrapolating the experimentally observed linear part the $M^{2}(T)$ dependence (see solid lines in Fig. 2b) one can estimate, based on the mean-field model, the ferromagnetic Curie temperature $T_{\mathrm{c}}$.

\section{Discussion and conclusions}

The analysis of the temperature dependence of the magnetization shows that the layers S2-S5 undergo the ferromagnetic transition with the Curie temperature 
in the range $T_{\mathrm{c}}=30-36 \mathrm{~K}$. The magnetic phase transition region is rather broad indicating the presence of some ferromagnetic correlations up about $T \approx 2 T_{\mathrm{c}}=$ 60-70 K. The diamagnetic background contribution due to $\mathrm{BaF}_{2}$ substrate becomes dominant, as expected, only at rather high temperatures $(T>100 \mathrm{~K})$. In the layer S1, which was grown with the highest Te flux applied, the high temperature ferromagnetic tail is observed on the $M(T)$ dependence indicating the presence of a second magnetic phase with the Curie temperature above $130 \mathrm{~K}$. The EDXRF analysis of the chemical composition of the layers revealed that in the samples S2-S5 the Te to Ge concentration ratio is in the range 1.02-1.11. This shows, as expected in these highly non-stoichiometric materials, an excess Te concentration (equivalent to the presence of Ge vacancies). In the sample $\mathrm{S} 1$ this ratio is substantially higher (about 1.32) which indicates the possibility of the formation during the growth of the S1 layer of germanium telluride alloys with Mn based not only on GeTe (standard case) but also on $\mathrm{Ge}_{2} \mathrm{Te}_{3}$ or $\mathrm{Ge}_{3} \mathrm{Te}_{4}$ tellurides with Te to Ge concentration ratio increased to $3 / 2$ or $4 / 3$, respectively. As the X-ray diffraction (XRD) investigations of the crystal structure of the (Ge,Mn)Te layers S1-S5 did not reveal any second crystal phase inclusions, the above mentioned idea remains a hypothesis only.

Figure 3 summarizes the influence of the stoichiometry conditions on the Curie temperature $T_{\mathrm{c}}$ of the $\mathrm{Ge}_{0.9} \mathrm{Mn}_{0.1}$ Te layers $\mathrm{S} 1-\mathrm{S} 5$. The broad magnetic transition region does not permit precise determination of the transition temperature. To reveal the experimentally observed trends the simple method of analysis was applied based on the $M^{2}(T) \propto\left(T-T_{\mathrm{c}}\right)$ dependence predicted by the mean field model for $T \leq T_{\mathrm{c}}$. The rather unusual $M(T)$ dependence found for layer $\mathrm{S} 5$ increases the experimental uncertainty of $T_{\mathrm{c}}$ determination.

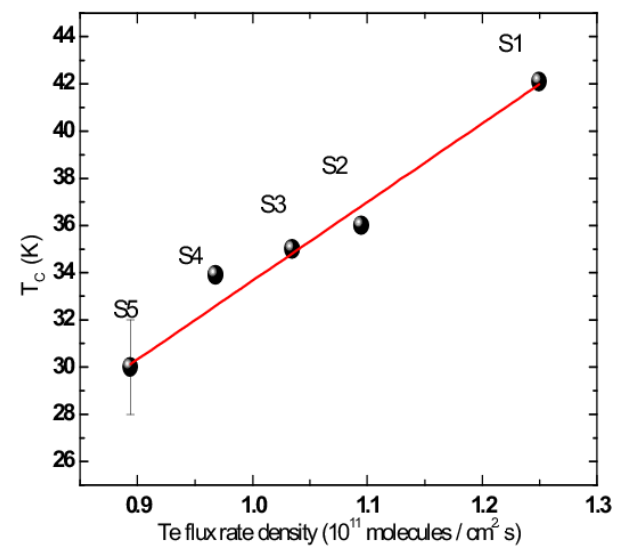

Fig. 3. The dependence of the ferromagnetic Curie temperature of $\mathrm{Ge}_{0.9} \mathrm{Mn}_{0.1}$ Te layers on Te flux applied during the epitaxial growth. 
The varying stoichiometry in samples S1-S5 also influences the magnetic field dependence of magnetization. At low fields, the magnetic moment at $T=5 \mathrm{~K}$ and $H=1 \mathrm{kOe}$ varies between 15 and $35 \mathrm{emu} / \mathrm{cm}^{3}$, as compared to $M_{\text {sat }}=86 \mathrm{emu} / \mathrm{cm}^{3}$ expected for the fully magnetically saturated system of substitutional $\mathrm{Mn}^{2+}$ ions in $\mathrm{Ge}_{0.9} \mathrm{Mn}_{0.1} \mathrm{Te}$ crystal with the lattice parameter about $0.6 \mathrm{~nm}$. This effect is particularly clearly observed for the layer S5 grown applying the lowest additional Te flux. For $\mathrm{Ge}_{0.9} \mathrm{Mn}_{0.1}$ Te layer grown under such technological conditions the full saturation at $T=5 \mathrm{~K}$ (i.e. about $1 / 6$ of $T_{\mathrm{c}}$ ) is not reached even at relatively high magnetic field of $70 \mathrm{kOe}$. These experimental findings can be explained considering the possible co-existence in (Ge,Mn)Te layers (grown under such technological conditions) of MnTe-rich and MnTe-poor regions at the microscale. This physico-chemical phase separation effect may be accompanied by a non-homogeneous distribution of conducting carriers expected to accumulate in the regions with lower Mn content due to the band gap difference [5]. Under such conditions the experimentally observed increase in the magnetization at high magnetic fields would correspond to the magnetization due to MnTe-rich regions with low carrier concentration and dominating antiferromagnetic short-range interactions. To fully magnetically saturate such material the magnetic fields in excess of $100 \mathrm{kOe}$ are known to be required. To substantiate this explanation a sophisticated, detailed analysis of Mn distribution at the micro-scale will be required, as successfully applied, e.g. in the case of Ge:Mn system [6].

In conclusion, we experimentally studied the magnetic properties of the epitaxial (Ge,Mn)Te layers grown by MBE technique on $\mathrm{BaF}_{2}$ (111) substrate. The layers with $\mathrm{Mn}$ content of 10 at.\% were grown with crystal stoichiometry controlled by varying additional Te molecular flux. The $\mathrm{Ge}_{0.9} \mathrm{Mn}_{0.1}$ Te layers undergo the ferromagnetic transition at the Curie temperature $T_{\mathrm{c}}=30-42 \mathrm{~K}$, depending on the growth conditions, with the temperature dependence of magnetization revealing a broad transition region. The magnetic field dependence of magnetization shows that to reach the full magnetic saturation in (Ge,Mn)Te layers the application of an external field higher that $70 \mathrm{kOe}$ is required. These experimental findings are discussed considering (Ge,Mn)Te layers as consisting of MnTe-rich (with lower hole concentration) and MnTe-poor (with higher hole concentration) micro-regions dominated by antiferromagnetic or ferromagnetic exchange interactions, respectively.

\section{Acknowledgments}

This work was supported by the research project No. 0992/T02/2007/32 granted for the period 2007-2010 by the Ministry of Science and Higher Education (Poland). 


\section{References}

[1] R.W. Cochrane, M. Plischke, J.O. Strom-Olsen, Phys. Rev. B 9, 3013 (1974).

[2] T. Story, in: Lead Chalcogenides - Physics and Applications, Ed. D.R. Khokhlov, Taylor and Francis, New York 2003, p. 385.

[3] W.J.M. de Jonge, T. Story, H.J.M. Swagten, P.J.T. Eggenkamp, Europhys. Lett. 17, 631 (1992).

[4] P.J.T. Eggenkamp, H.J.M. Swagten, T. Story, V.I. Litvinov, W.J.M. de Jonge, Phys. Rev. B 51, 15250 (1995).

[5] Y. Fukuma, H. Asada, M. Arifuku, T. Koyanagi, Appl. Phys. Lett. 80, 1013 (2002).

[6] W.Q. Chen, K.L. Teo, S.T. Lim, M.B.A. Jalil, T. Liew, T.C. Chong, Appl. Phys. Lett. 90, 142514 (2007).

[7] Y. Fukuma, K. Goto, S. Senba, S. Miyawaki, H. Asada, T. Koyanagi, H. Sato, J. Appl. Phys. 103, 053904 (2008).

[8] W. Knoff, P. Dziawa, V. Osinniy, B. Taliashvili, V. Domuchowski, E. Łusakowska, K. Swiatek, T. Story, Mater. Sci. — Poland 25, 295 (2007). 